\title{
ProteÇÃo DE MOtores Veiculares duRANTE ENSAIOS EM DinAMôMETRO DE MOTOR
}

\author{
Robson Baptista Gama ${ }^{1}$ e Paulo César de Ferreira Gomes ${ }^{1}$ \\ ${ }^{1}$ Robert Bosch Ltda \\ E-mails: robson.gama@br.bosch.com,paulo.gomes@br.bosch.com
}

\section{RESUMO}

Frente aos novos desafios decorrentes do novo regime automotivo INOVAR AUTO [1], a busca por motores que proporcionem um consumo reduzido de combustível indica que a utilização de motores DI (Direct Injection) aliados à tecnologia Flex-Fuel será mais comum no Brasil nos próximos anos. Como consequência, os requisitos para ensaio destes novos motores serão mais severos em relação à segurança e ao custo, exigindo o desenvolvimento contínuo dos sistemas de medição de motores.

No desenvolvimento de um sistema de gerenciamento de motor veicular é imprescindível a utilização de um dinamômetro de motor de forma a simular diferentes situações de regime de trabalho, inclusive aquelas que expõem o motor às situações críticas, podendo danificálo. Sabendo deste agravante, os mais variados controles de dinamômetro possuem configurações de alarmes de segurança, que monitoram o motor e garantem o seu funcionamento seguro. Entretanto, alguns sistemas não possuem uma resposta rápida e efetiva para atuar em eventos de alta frequência, ou ainda, sistemas mais sofisticados impõe a penalidade de um custo muito elevado.

Este trabalho tem como objetivo apresentar um dispositivo de segurança de baixo custo que possibilita interromper o fornecimento de combustível ao motor assim que picos de pressão no cilindro forem detectados.

\section{INTRODUÇÃO}

Através do Decreto Lei 7819, de 03/10/2012, o governo brasileiro lançou o programa INOVAR AUTO que representa um grande desafio para as montadoras de veículos automotivos comercializados no Brasil. O novo programa estimula a inovação e a competitividade, podendo resultar em benefícios diretos para a sociedade, indústria e governo. "Ter tecnologias mais limpas, veículos mais econômicos, mais geração de empregos e ampliar a competitividade de nossos carros no cenário mundial é bom para todos." [2];

Entre os objetivos do novo regime, destaca-se a redução do consumo de combustível e das emissões de gás carbônico, visando o desenvolvimento de tecnologias sustentáveis no ramo automobilístico. Neste cenário, enquadram-se a Tecnologia Flex-Start, o Sistema Flex-Fuel de injeção direta de combustível (DI-systems), Start-Stop e Downsizing, todas estas 
desenvolvidas pela Bosch para atingir os desafios de redução das emissões de poluentes e promover a economia de combustível.

Para o emprego e desenvolvimento destas tecnologias, faz-se necessário a utilização de bancadas de teste com recursos de segurança especiais. Entre eles encontra-se o Sistema de Segurança para Proteção de Motores Veiculares durante Ensaios em Dinamômetro de Motor, descritos neste artigo.

\section{ECONOMIA DE COMBUSTÍVEL COM MOTORES DI}

Uma das tendências do novo programa INOVAR AUTO será alavancar a utilização de novos motores no mercado brasileiro, como, por exemplo, aqueles com o Sistema Flex-Fuel de injeção direta de combustível (DI-systems). Isto porque uma de suas principais vantagens é a redução de consumo de combustível. Enquanto em motores PFI (Port Fuel Injection) existe uma quantidade significativa de gasolina não queimada devido à mistura ar/combustível se formar fora da câmara de combustão, nos motores DI o controle preciso do combustível injetado e do ar admitido no interior da câmara de combustão resultam em uma queima mais efetiva, garantindo baixos níveis de emissão de gases.

Além disso, quando utilizando a tecnologia Flex-Fuel, é possível explorar nos motores DI taxas de compressão mais elevadas para obter valores de torque superiores. Contudo, durante os ensaios de desenvolvimento do motor veicular, é necessário garantir que os limites estruturais do protótipo ensaiado não sejam ultrapassados devido às altas temperaturas e pressões de trabalho na câmara de combustão, sendo necessário sistemas de monitoramento dos testes mais rigorosos e que possam atuar em frequências elevadas para proteção de tais motores.

\section{ALARMES DE PROTEÇÃO EM DINAMÔMETRO DE MOTOR}

Existem diversos modelos de dinamômetros de motor, bem como vários softwares para operação destas máquinas. Em quase todos existem sistemas de segurança que monitoram determinadas grandezas do motor ensaiado. Assim, é possível diagnosticar possíveis falhas no sistema, erros de operação e até condições acima dos limites especificados para o protótipo em teste.

Quando um alarme é acionado, existem alguns recursos que o sistema pode utilizar para manter o motor em níveis seguros de operação, o qual possui um alto custo por se tratar de um protótipo. Dentre eles, destacam-se:

- Atuação direta no sistema de controle do dinamômetro, através de uma rotina automática. Neste caso, normalmente o motor ensaiado é limitado à operação em marcha lenta. A circulação de água e óleo é mantida e os valores de pressão e temperatura na câmara de combustão são reduzidos a níveis seguros. 
- Atuação no sistema de gerenciamento do motor, através do enriquecimento da mistura ar/combustível e/ou alteração do ponto de ignição. Este caso é muito comum para o controle de alguns fenômenos de combustão anormal [3, 4].

- Atuação drástica no sistema de controle ou motor, desligando-o completamente.

Entretanto, tais configurações de alarme possuem limites quanto ao tempo de reação em sistemas mais simples, e nem sempre são capazes de proteger o objeto de ensaio frente aos eventos de alta frequência. Ou ainda, a utilização de sistemas com controles mais sofisticados está associada a custos elevados devido à complexidade de sua construção.

Considere um exemplo: um determinado motor está operando em um ciclo contínuo a 6000 rpm, em regime de plena carga (corpo de borboleta totalmente aberto). A temperatura do catalisador está sendo monitorada por um termopar que está ligado ao controle do dinamômetro de motor. O sistema está configurado de forma que, caso a temperatura medida pelo sensor ultrapasse um determinado valor, automaticamente uma rotina de segurança é iniciada, abortando o ensaio. A rotação do motor é reduzida para marcha lenta (entre 800 e $1000 \mathrm{rpm}$ ), para que a temperatura do catalisador diminua.

No exemplo acima, o sistema de alarme é efetivo. Não existem prejuízos para o motor, nem para o dinamômetro. O tempo de resposta é suficiente para resfriar o motor a níveis seguros e preservar o catalisador. Ainda é possível alterar o tempo de resposta ou mesmo modificar o limite de alarme conforme as especificações desejadas.

Considere agora um segundo exemplo, conforme será abordado neste trabalho. As mesmas configurações anteriores são adotadas: $6000 \mathrm{rpm}$, plena carga. Os mapas de calibração do sistema de gerenciamento do motor são alterados seguindo um procedimento préestabelecido, orientado pela leitura de diversos sensores instalados no motor. Entre eles, existem alguns que monitoram a pressão na câmara de combustão, já que o protótipo possui limites estruturais que não podem ser ultrapassados. Tais sensores também são capazes de detectar as combustões anormais que ocorrem no cilindro, as quais são prejudiciais ao protótipo podendo levar à destruição do mesmo conforme mostrado na Figura 1.

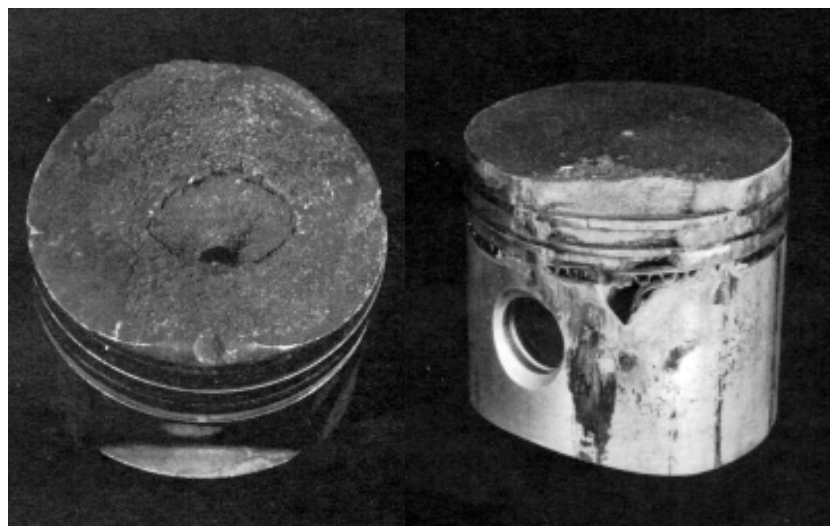

Figura 1 - consequência de pré-ignição em motores veiculares [5]

Seja pela aparição dos fenômenos de detonação e/ou pré-ignição, seja pelas condições extremas de operação, o motor é exposto a elevados picos de pressão. Como no primeiro exemplo, é possível controlar este problema monitorando a pressão na câmara de combustão e 
programando um limite conforme especificação do motor. Caso o limite seja ultrapassado, o sistema de controle do dinamômetro de motor atua desligando ou limitando o funcionamento do motor. O problema estaria sanado se não fosse à frequência de trabalho dos motores de combustão interna. Analisando um motor ciclo Otto de 4 tempos (no qual o sistema de proteção proposto é utilizado), existem quatro fases no ciclo de funcionamento: admissão, compressão, combustão e escape.

Um ciclo completo em um único cilindro tem a duração de duas voltas (720 graus do eixo de manivelas). Isso significa que para um motor de quatro cilindros, a cada meia volta (180 graus) existe uma combustão em um dos quatro cilindros. Significa que em 1,0 segundo, para uma velocidade do motor de $6000 \mathrm{rpm}$, acontecem cerca de 200 combustões no motor. Ou seja, a cada $5 \mathrm{~ms}$ aproximadamente ocorre uma combustão. Assim, mesmo que o controle do dinamômetro reagisse em apenas 1 segundo, significaria permitir mais de 200 combustões no motor com níveis de pressão altíssimos. Certamente o motor seria danificado.

A Figura 2 mostra os diagramas de pressão na câmara de combustão durante a ocorrência de uma mega detonação que pode levar à destruição imediata do pistão conforme mostrado na Figura 1.

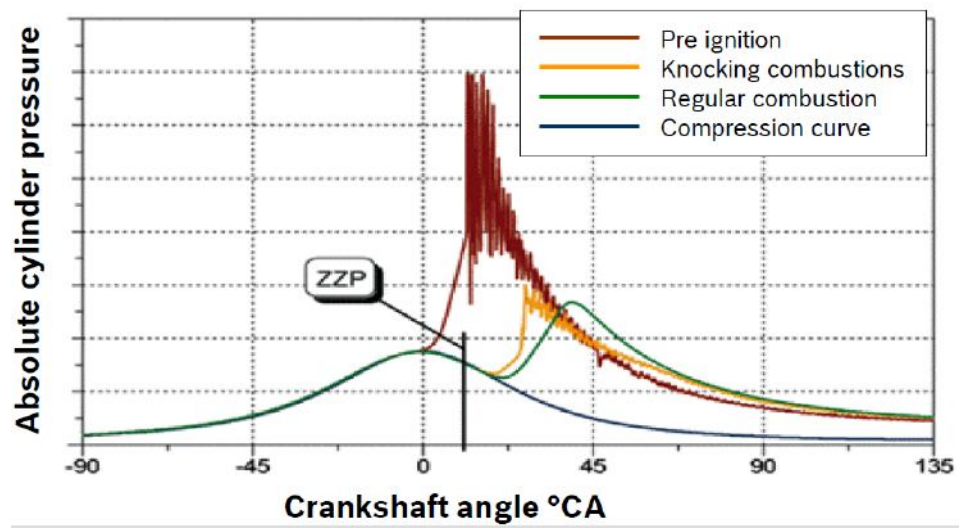

Figura 2 - curva de pressão: típico evento de pré-ignição [6]

\section{SISTEMA DE SEGURANÇA COM ACIONAMENTO RÁPIDO}

Sabendo da necessidade de um sistema de segurança que proporcione um tempo de resposta menor que 1 milissegundo, é proposto um dispositivo controlado por portas lógicas. Sua construção (semicondutores) permite um regime de trabalho na ordem de $10 \mathrm{~ns}$, além de possuir um baixo custo.

De forma sucinta, o sistema é composto por um acoplador óptico [7], um flip-flop [8] e um relê para chaveamento [9], além de uma entrada de sinal de trigger. Este é enviado por um equipamento que monitore a pressão no cilindro do motor conforme mostrado na Figura 3. 


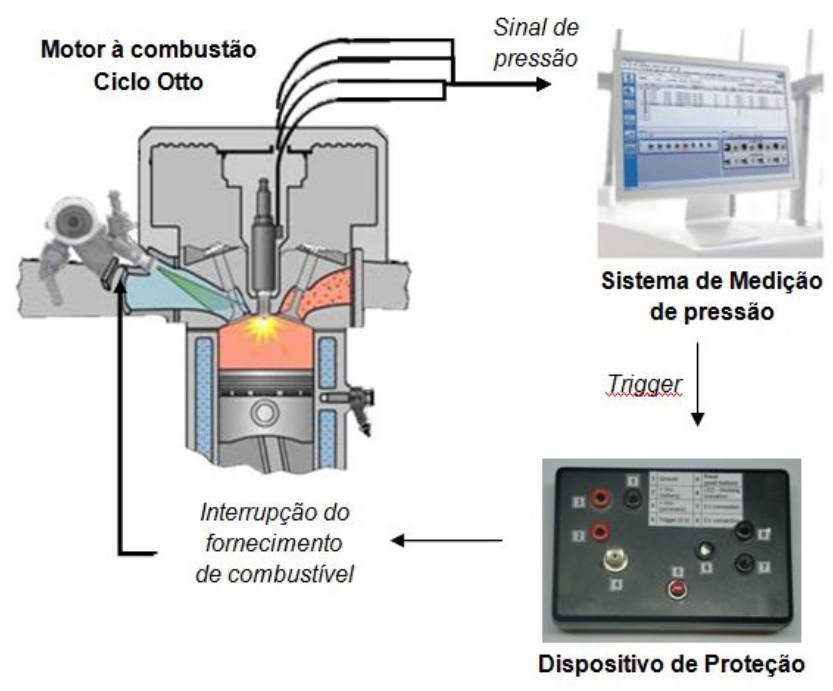

Figura 3 - Diagrama de blocos do sistema

\subsection{SISTEMA DE MEDIÇÃO DE PRESSÃO}

Existem vários sistemas utilizados para monitorar a pressão nos cilindros do motor, sendo que alguns ainda permitem o acoplamento direto com o sistema de controle do dinamômetro de motor. A escolha do sistema utilizado deve seguir os critérios de leitura e processamento de dados que se queira obter das grandezas medidas.

Neste trabalho, um dos critérios de escolha do sistema de medição de pressão foi o tempo de resposta em função do sinal de trigger. Por isso, foi utilizado o sistema de medição da AVL, o IndiCom [10], o qual possibilita uma reação de acordo com a rotação do motor, na proporção de 1/RPS (rotações por segundo). Ou seja, ao identificar um pico de pressão no cilindro maior que o valor configurado como limite, para uma rotação de $6000 \mathrm{rpm}$, o sinal de $5 \mathrm{~V}$ será transmitido em $10 \mathrm{~ms}$. Isso significa permitir mais duas injeções nos cilindros subsequentes, além daquela que ocasionou um pico de pressão identificado pelo sistema.

Na Figura 4 é possível visualizar um típico gráfico de medição de pressão obtido através do IndiCom. Devido a sua complexidade e variedade de funções, este sistema (assim como outros similares) possui algumas opções de alarme que também podem ser utilizadas para monitoramento e controle do protótipo em teste.

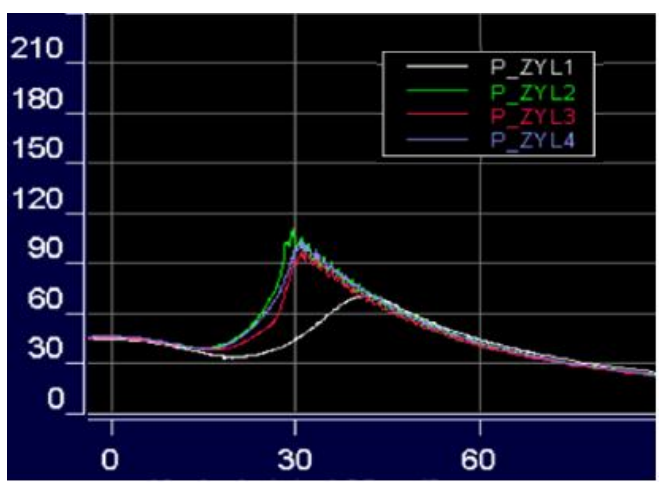

Figura 4-Gráfico de medição de pressão [11] 


\subsection{SISTEMA DE CONTROLE}

O sistema de controle é constituído principalmente por um circuito lógico (flip flop) que possibilita um tempo de resposta na ordem de $10 \mathrm{~ns}$.

Na Figura 5 é possível visualizar o esquema elétrico proposto. O circuito pode ser dividido em duas partes: bloco de alimentação e entrada de sinal (trigger), e bloco de controle e indicação de funcionamento.

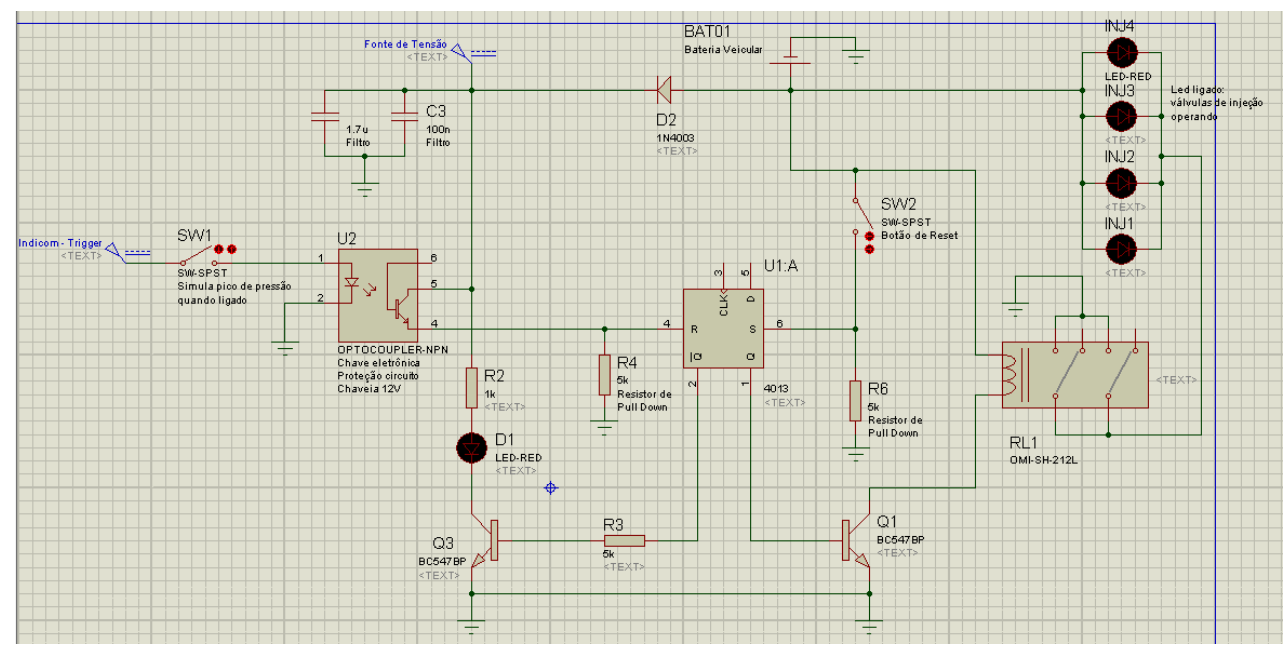

Figura 5 - Esquema elétrico do sistema de segurança

\subsubsection{Bloco de alimentação e entrada de sinal}

Para possibilitar a construção de um sistema portátil, o dispositivo possui dois tipos de entrada de alimentação: a primeira através de fonte de tensão estabilizada; e a segunda através de uma bateria veicular. Foram utilizados capacitores eletrolíticos para filtrar o sinal de entrada (eliminando a tensão de ripple - ondulação devido à corrente alternada) e um diodo 1N4003, o qual impede a circulação da corrente da fonte de tensão no sentido da bateria. Com isso, além da aplicação do sistema em dinamômetro de motor, é possível ainda estender sua utilização em veículos automotivos.

Além de alimentar o circuito, a tensão fornecida pela fonte ou bateria servirá de entrada para o flip flop, o qual possibilita uma lógica de controle em $12 \mathrm{~V}$.

O sistema de segurança atua proporcionalmente ao sinal de entrada proveniente do sistema de medição de pressão. Este, conforme ilustrado na Figura 4, monitora o sinal de pressão dos cilindros da câmara de combustão. Nele é possível configurar um sinal de trigger correspondente a um limite de pressão. Assim, caso o valor de pressão exceda o valor determinado, um sinal de $5 \mathrm{~V}$ será enviado ao circuito lógico.

O acoplador é responsável por chavear a tensão de alimentação para o flip flop. De forma sucinta, este componente recebe o sinal de $5 \mathrm{~V}$ e aciona um 
led interno, emitindo luz na direção da base do foto-transistor para polarizálo, funcionando como uma chave óptica. Este, por sua vez, conduz a corrente proveniente da alimentação do circuito (12V) no sentido de uma das entradas do circuito lógico.

\subsubsection{Bloco de controle e indicação de funcionamento}

Neste bloco estão localizados os componentes mais importantes do sistema de segurança. São eles:

- relê miniatura HE722A1210 (contato NF). Componente responsável por interromper a conexão das válvulas de injeção, com capacidade de contato até $200 \mathrm{~V}$. Assim, possibilita a atuação tanto em motores do tipo PFI quanto em motores DI.

- Flip flop CD4013BE. É responsável por interpretar o sinal proveniente do trigger e acionar o relê.

Ao receber um sinal $12 \mathrm{~V}$ na entrada RESET (chaveado pelo acoplador ótico), o CI disponibiliza uma saída de igual potencial na saída Q (nível lógico 1), polarizando o transistor e por fim acionando o relê. A saída $\bar{Q}$, com funcionamento inverso (nível lógico 0), irá polarizar o respectivo transistor ligado a ela para energizar o led de indicação do sistema. Assim, quando o led estiver aceso, o circuito está atuando (alimentação da válvula de injeção interrompida).

Pode-se ainda prover um sinal 12V (através do botão RESET - chave do tipo push-bottom) na entrada SET do flip flop. Quando isso acontecer, existem duas possibilidades:

- quando o sinal em RESET for igual a $0 \mathrm{~V}$, as saídas Q e $\overline{\mathbf{Q}}$ terão nível lógico 1 e 0 , respectivamente. Isto fará com que o relê volte a sua condição natural (normalmente fechado), e o led permanecerá apagado.

- quando existir um sinal $12 \mathrm{~V}$ em RESET, as saídas Q e $\overline{\mathrm{Q}}$ serão 0 e 1 , respectivamente. Ou seja, como ainda existe um valor proveniente do trigger, não é possível permitir o funcionamento das válvulas de injeção.

Portanto, sempre que houver um valor de pressão superior ao trigger configurado (seja originado por uma condição extrema, seja por fenômenos de detonação ou pré-ignição, ou ainda por condições anormais de funcionamento), haverá um sinal de $5 \mathrm{~V}$ na entrada do sistema de segurança. O relê passará de NF para NA, interrompendo a alimentação das válvulas de injeção. 
Tabela 1 - Tabela verdade CD4013BE (trigger $x$ relê)

\begin{tabular}{|c|c|c|c|c|c|c|c|}
\hline \multirow{2}{*}{\multicolumn{2}{|c|}{ CD4013BE }} & \multicolumn{2}{|c|}{ Entrada } & \multicolumn{2}{|c|}{ Saída } & \multirow{2}{*}{$\begin{array}{c}\text { Relê } \\
\text { (contato) }\end{array}$} & \multirow{2}{*}{ LED } \\
\hline & & RESET & SET & $Q$ & $\overline{0}$ & & \\
\hline \multirow{5}{*}{ Trigger } & \multirow{3}{*}{ OV } & 0 & 0 & $Q$ & $\overline{\mathrm{Q}}$ & Aberto* & Aceso \\
\hline & & 0 & 0 & Q & $\overline{\mathrm{Q}}$ & Fechado ${ }^{\star *}$ & Apagado \\
\hline & & 0 & $12 \mathrm{~V}$ & 1 & 0 & Fechado & Apagado \\
\hline & \multirow{2}{*}{$5 \mathrm{~V}$} & $12 \mathrm{~V}$ & 0 & 0 & 1 & Aberto & Aceso \\
\hline & & $12 \mathrm{~V}$ & $12 \mathrm{~V}$ & 1 & 1 & Fechado $^{* * *}$ & Aceso \\
\hline
\end{tabular}

* ao energizar o circuito com a fonte ou bateria

${ }^{* *}$ circuito já em funcionamento

${ }^{* * *}$ relê fechado apenas enquanto push bottom acionado (SET $=12 \mathrm{~V}$ )

Sem combustível, não haverá combustão no cilindro, fazendo com que a pressão na câmara seja reduzida a níveis muito baixos, eliminando a situação de risco.

\section{RESULTADOS}

A Figura 6 apresenta o hardware do sistema de segurança proposto.

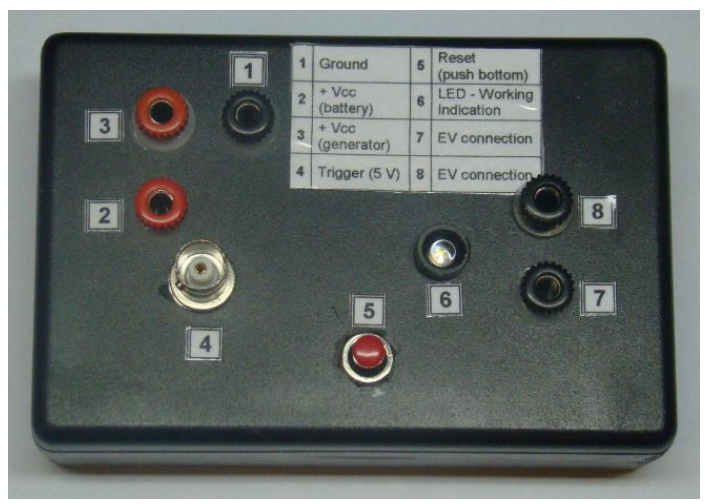

Figura 6 - Sistema de Segurança

Para validar o seu funcionamento, primeiramente foram realizados os testes em bancada. Utilizou-se uma fonte de tensão de duas saídas, ajustada em $12 \mathrm{~V}$ e $5 \mathrm{~V}$, para alimentação e simulação do trigger respectivamente. Foi comprovado o comportamento descrito na Tabela 1 medindo a continuidade das conexões que interrompem o contato das válvulas de injeção.

Em seguida foi realizado um ensaio em um motor veicular do tipo PFI acoplado ao dinamômetro. O fio comum das válvulas de injeção foi interrompido, colocando o sistema em série. A saída de trigger do sistema de medição de pressão foi conectada à entrada do sistema de segurança, o qual foi energizado com $12 \mathrm{~V}$, proveniente de uma fonte de tensão estabilizada. Como descrito na Tabela 1, sempre que o sistema é energizado, o circuito atua de tal forma que o relê seja acionado. Portanto, o botão de reset foi acionado. 
Para obter o sinal de pressão da câmara de combustão, o protótipo foi instrumentado com velas de ignição Kistler modelo 6117 [12], as quais foram conectadas ao sistema de medição de pressão IndiCom (AVL). Este, por sua vez, foi configurado para medição de pressão na câmara de combustão, limitado por um trigger, o qual garantia o funcionamento do motor dentro de seus limites estruturais de pressão máxima na câmara de combustão.

O motor foi condicionado de forma a obter uma pressão no cilindro superior à pressão configurada no trigger. Foi constatado um sinal de 5V na saída do IndiCom e na entrada do sistema de segurança, o qual interrompeu a conexão das válvulas de injeção em um tempo menor que 0,2ms, como ilustra a Figura 7.

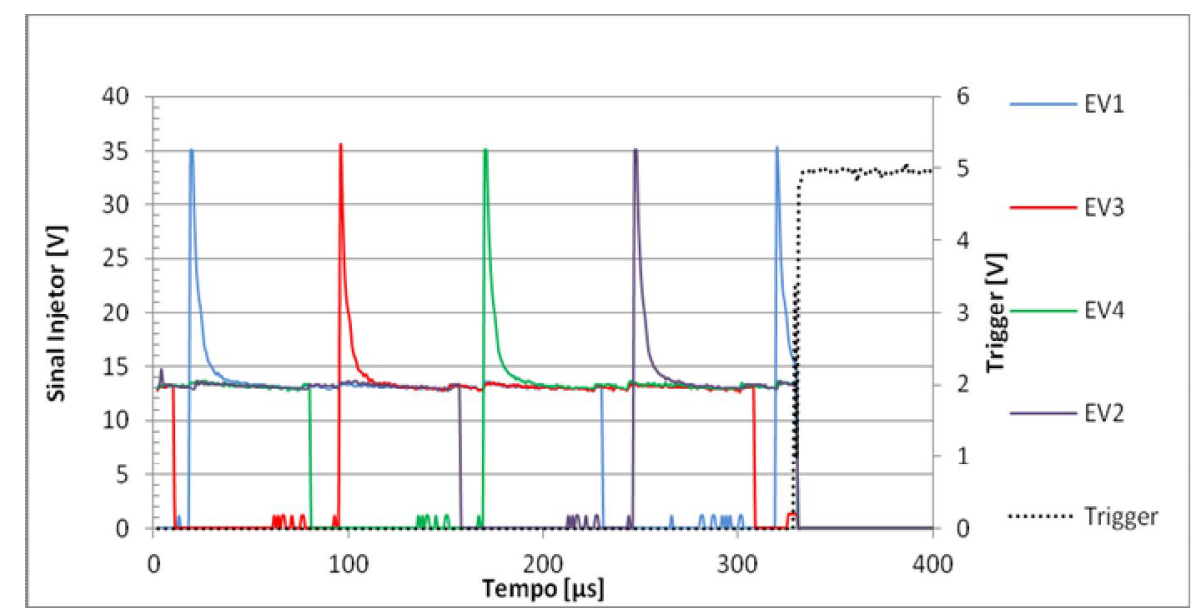

Figura 7 - tempo de resposta do sistema de segurança em motor veicular utilizando relê para chaveamento

Repetiu-se a mesma operação para 12 pontos distintos, variando as condições de rotação e carga. Em todas as situações o sistema mostrou-se eficiente, interrompendo o fornecimento de combustível antes do ciclo de queima do cilindro subsequente.

Porém, após os ensaios, levantou-se a seguinte questão: qual a influência do sistema de segurança devido ao aumento da resistência elétrica no circuito das válvulas de injeção? Sabese que as válvulas de injeção em motores do tipo PFI possuem uma resistência elétrica maior que àquelas utilizadas em motores de injeção direta. Isso porque, devido à alta pressão em que estas operam, é necessária uma energia maior para abrir e fechar a válvula, necessitando de um valor de tensão alto. Enquanto as válvulas de motores PFI operam em 12V, estas podem operar em valores de pico de até $70 \mathrm{~V}$. O sistema de segurança proposto apresentou valores de resistência elétrica na ordem de $0,05 \Omega$. Significa que, para motores do tipo PFI, a resistência do circuito não impactará no tempo de abertura/fechamento da válvula. Mas para motores DI, existe a possibilidade de o sistema influenciar no ciclo de injeção, como mostra a Figura 8. As linhas pontilhadas correspondem ao impacto devido ao aumento da resistência elétrica. 


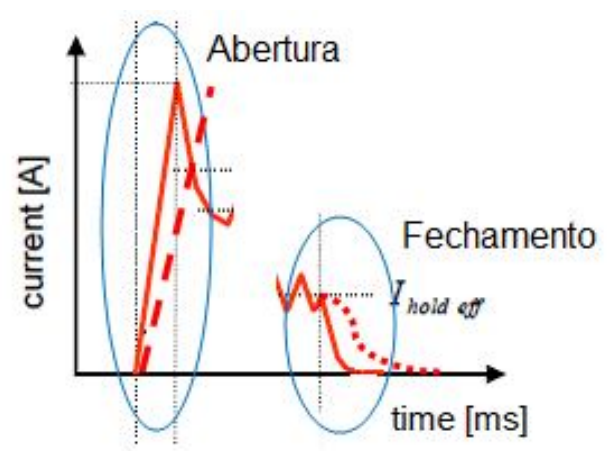

Figura 8 - Impacto nos tempos de abertura e fechamento do injetor devido ao aumento de resistência no circuito de injeção. [13]

Significa que, quando o circuito estiver em série com as válvulas, o sistema de gerenciamento do motor estará submetido a uma resistência maior do que a de projeto, podendo atrasar o ciclo de injeção. Além disso, a abertura do relé com uma carga indutiva (injetores) irá gerar arco e consequentemente danificar os contatos com o tempo, aumentando sua resistência.

Para evitar este impasse, propõem-se a utilização de transistores do tipo MOSFET no chaveamento do circuito dos injetores. Esses possuem uma resistência elétrica $\left(R_{d s o n}\right)$ na ordem de miliohm. Um modelo sugerido seria o STP190N55LF3, com uma resistência estática máxima de $4,5 \mathrm{~m} \Omega$.

\section{CONCLUSÃO}

O Programa INOVAR AUTO, decretado pelo governo brasileiro em 2012, estabelece nos próximos 5 anos um grande desafio para as montadoras de veículos automotivos comercializados no Brasil. Para atingir as metas de redução de emissões de poluentes e aumentar a eficiência energética dos seus motores, a indústria automobilística deverá utilizar bancadas de teste com recursos de segurança especiais, assim como mostrado neste artigo.

O Sistema de Segurança aqui descrito mostrou-se eficiente, operando com um tempo de reposta que garante a integridade do protótipo ensaiado, proporcionando maior segurança no processo de desenvolvimento e aplicação de um motor veicular.

Uma vez que o sistema foi desenvolvido a partir de componentes e princípios de funcionamento relativamente simples e de fácil instalação/manutenção, este possui um alto valor agregado com baixo custo de implementação. Esta é uma das principais vantagens do projeto, já que outros sistemas e dispositivos semelhantes possuem um custo elevado, e demandam um grande esforço para configuração e montagem.

O projeto também tem potencial para o controle de motores de injeção direta, desde que o aumento de resistência elétrica no circuito de injeção não tenha impacto nos tempos de abertura e fechamento da válvula de injeção. E mesmo que isso aconteça, a substituição dos relês por transistores do tipo MOSFET indicam a garantia de uma resistência elétrica quase nula, não afetando a resposta das válvulas injetoras. Além disto, o MOSFET tem potencial para diminuir o tempo de atuação do Sistema de Segurança, aumentando a proteção do protótipo. 


\section{REFERÊNCIAS}

[1] MDIC. Decreto estabelece as regras do Inovar-Auto, novo regime automotivo brasileiro. Disponível em: http://www.desenvolvimento.gov.br/sitio/interna/noticia.php?area=1\&noticia $=11857$. Acesso em 25 de março de 2013.

[2] PASQUINI, Jair. Inovar Auto estimula soluções para eficiência energética dos carros brasileiros. Conexão CaP, Campinas, Edição 1, página 12, Abril 2013.

[3] ROGERS, David R. Engine Combustion: Pressure Measurement and Analysis. Warrendale, EUA: SAE International, 2010.

[4] HEYWOOD, John B. Internal Combustion Engine Fundamentals. Singapore: McGraw-Hill International Editions, 1989.

[5] GUENTHER, Jeffrey. Detonation and Pre-Ignition. Hastings, USA, Hastings Manufacturing Company, 2008.

[6] BODO, Oliver. Motronic Training Document - Pre-Ignition. Version 1.0 - 03/2013, Robert Bosch GmbH.

[7] HEWLETT PACKARD. Very High CMR, Wide VCC Logic Gate Optocouplers. Catálogo técnico. Disponível em: http://www.datasheetcatalog.net/pt/datasheets_pdf/H/C/P/L/ HCPL-2201.shtml. Acesso em 21 de janeiro de 2013.

[8] NATIONAL SEMICONDUTOR CORPORATION. Datasheet do componente CD4013BM/CD4013BC Dual D Flip-Flop. Catálogo técnico. Disponível em: http://www. alldatasheet.com/datasheet-pdf/pdf/26848/TI/CD4013BE.html. Acesso em 21 de janeiro de 2013.

[9] HAMLIN. HE700 D.I.L. Relay Features and Benefits. Catálogo técnico. Disponível em: http://octopart.com/datasheet/he722a1210-hamlin-694247-13059395. Acesso em 21 de janeiro de 2013.

[10] AVL LIST GMBH. AVL Advanced Combustion Analysis Software. Catálogo técnico. Disponível em: https://www.avl.com/indicom-indicating-software. Acesso em 13 de março de 2013.

[11] BIEHL, Matthias; BODO, Oliver. Pre-Ignition in Highly Boosted Turbo Engines. Schwieberdingen, DE. Robert Bosch GmbH, DGS-EC/EAU2, 2011.

[12] KISTLER INSTRUMENTE AG. Measuring Spark Plug with Integrated Cylinder Pressure Sensor. Catálogo técnico. Disponível em: http://www.kistler.com/us/en/support/ downloads/datasheets. Acesso em 13 de março de 2013.

[13] GENTINI, Isaac. Informações sobre válvula de injeção direta. [mensagem pessoal]. Mensagem recebida de < Isaac.Gentini2 @ br.bosch.com >, 03/04/2013. 